\title{
Recurrent perivascular inflammation induced by lipopolysaccharide (endotoxin) results in the formation of atheromatous lesions in vivo
}

\author{
Markus Georg Engelmann ${ }^{1}$, Constanze Veronika Redl ${ }^{1}$ and Sigrid Nikol ${ }^{1,2}$ \\ ${ }^{1}$ Medical Department I, Klinikum Grosshadern, Ludwig Maximilian University, Munich, Germany and \\ ${ }^{2}$ Department of Cardiology and Angiology, Universitätsklinikum, University of Münster, Germany
}

\begin{abstract}
Bacteria and viruses are suspected to induce arteriosclerosis; however, most investigators have focused on coincidences rather than causal relationships. The aim of this work was to establish a rabbit model in which the vessel reaction to local perivascular injection of defined bacterial products can be analyzed. A total of 23 rabbits were randomized to four groups. Groups $A$ and $B$ were fed a normal diet, groups $C$ and $D$ were fed a cholesterolenriched diet. Groups A and C were treated with a single perivascular injection of bacterial lipopolysaccharide (LPS, endotoxin) placed next to auricular, carotid and femoral arteries, and sodium chloride placed next to the contralateral arteries (control). Group B and D animals were treated with repeated perivascular injections over 90 days. Vascular tissues $(n=116$ treated segments of 23 rabbits) were analyzed using morphometry at histology, and using immunohistochemistry to detect macrophages, lymphocytes and vascular smooth muscle cells. LPS treatment resulted in transient focal intima thickening. After single LPS application, no increase in atheromatous lesion formation was observed in comparison with controls (group C, lesion area index $0.031 \pm 0.012$ vs $0.015 \pm 0.006, P=1.0$ ). Repeated LPS application resulted in significant atheromatous lesion formation compared with saline control (group $D$, lesion area index $0.148 \pm 0.049$ vs $0.008 \pm 0.006, P=0.003$ ) in hypercholesterolemic rabbits. Repeated LPS inflammation in normocholesterolemic did not lead to atheromatous lesion formation (intima media ratio $0.04 \pm 0.01$ vs $0.04 \pm 0.007, P=1.0$ ). Single perivascular administration of low-dose bacterial LPS resulted in transient focal intimal thickening, while significant increase in lesion formation occurred after repeated LPS application in cholesterol-fed animals. In conclusion, this animal model will allow the assessment of the impact of defined dosages of different bacterial pathogens onto the vascular wall in the context of atherogenesis. The atheromatous lesion-promoting effect of repeated perivascular administration of LPS supports the hypothesis that bacterial pathogens may be involved in atherogenesis.
\end{abstract}

Laboratory Investigation (2004) 84, 425-432, advance online publication, 16 February 2004; doi:10.1038/labinvest.3700065

Keywords: atherosclerosis; inflammation; animal model; hypercholesterolemia; lipopolysaccharide; endotoxin

Inflammation is thought to play an important role in atherosclerosis and its acute complications. Adhesion of monocytes to vascular endothelium and their migration into the subendothelial space of arterial wall has been suggested to be an early event in the development of atherosclerotic plaques. ${ }^{1}$ Increased adhesion and migration of various types of leukocytes, including monocytes, lymphocytes and neutrophils, is associated with conditions where there is an increased risk of atherosclerosis, for example hypercholesterolemia. Infiltration of leukocytes occurs into all layers of the vessel wall.

Correspondence: MG Engelmann, MD, Medical Department I, Klinikum Grosshadern, Marchioninistr. 15, Munich 81377, Germany. E-mail: markus.engelmann@med.uni-muenchen.de

Received 24 June 2003; revised 22 October 2003; accepted 29 October 2003; published online 16 February 2004
Infectious episodes, which may lead to leukocyte infiltration, including infections with Gram-negative bacteria, may be involved in the pathogenesis of atherosclerosis. ${ }^{2,3}$ Lipopolysaccharide (LPS or endotoxin) is an important component of the cell wall of Gram-negative bacteria. LPS has numerous biological activities and is responsible for many pathological conditions caused by Gram-negative bacteria. Recent studies have suggested that LPS-induced immune mechanisms may be important in the atherosclerotic process. ${ }^{4-6}$ A recently described polymorphism in the gene for the LPS receptor CD14 is associated with myocardial infarction in patients with a low atherosclerotic risk profile, thus implicating bacterial LPS in atherogenesis and/or its complications in humans. ${ }^{7}$

The purpose of the present investigations was to assess the effect of perivascular administration of 
Escherichia coli LPS to the vessel wall in a rabbit model, under conditions of normocholesterolemia and hypercholesterolemia. A perivascular rather than systemic approach was chosen to avoid septicemia due to systemic contamination, leading to premature animal death as described by other groups. ${ }^{8}$ In addition, controlled local application of defined dosages allows for the assessment of atherogenesis in predefined arterial segments that are not predisposed to atherosclerosis. Creation of a model in which a transient focal inflammatory response can be generated would allow systematic evaluation of the influence of various bacterial products, for example, Chlamydia pneumoniae on lesion formation and plaque induction.

\section{Materials and methods}

\section{Animal Study Protocol}

A total of 23 female New Zealand White rabbits, mean weight $3.7 \pm 0.4 \mathrm{~kg}$, were investigated. Of these, 17 rabbits were randomly assigned to one of four groups. Two groups of rabbits (group A, $n=4$ and group $\mathrm{B}, n=5$ ) were fed a standard diet, group $\mathrm{C}$ animals $(n=4)$ were fed an atherogenic diet supplement including $0.5 \%$ cholesterol for 10 weeks. Four animals (group D) were fed a prolonged cholesterol diet consisting of 16 weeks of feeding. Additional six rabbits on the standard diet were used to test the concentration of LPS and for the assessment of the vessel reaction at early time points. These were killed on days 1, 6 or 18. Figure 1 shows details of the animal study protocol.

Rabbits in groups A and C, as well as the rabbits for the early time point experiments received a single perivascular injection of LPS ( $1 \mu \mathrm{g}, E$. coli strain J5, Rc mutant, Sigma) given locally to the auricular artery, the carotid and the superficial femoral artery, using $1 \mathrm{ml}$ syringes and 25-gauge needles. The injection next to the auricular artery was controlled under direct vision; the injections administered locally to the carotid and femoral arteries were guided by an $8 \mathrm{MHz}$ Doppler ultrasonic probe (Kranzbühler, Solingen, Germany). A volume of $100 \mu \mathrm{l}$ was administered next to the auricular artery; the other vessels received $200 \mu \mathrm{l}$ total volume. Each injection contained $1 \mu \mathrm{g}$ LPS. Contralateral arteries in each animal were treated with an equal volume of sodium chloride (0.9\%). Rabbits in groups B and D received three repeated LPS and control injections 4 weekly (group D animals at weeks 3,7 and 11 of cholesterol feeding). Group C rabbits received LPS injections 5 days following the onset of cholesterol-enriched diet. Investigations were carried out with the permission of federal government, conforming with national and local University guidelines on animal experiments.

\section{Histopathological Studies}

Rabbits were killed using intravenous pentobarbital. Carotid, superficial femoral, auricular arteries and aortas were removed, preserving surrounding adventitial tissue. After division into transversal segments, vessels were embedded in OCT (Sakura, Zoetervoude, NL) and snap-frozen in liquid nitrogen. Sections $(10 \mu \mathrm{m})$ were stained with hematoxylin and eosin, and van Giesson's elastic staining. Four segments were stained per treated site, and the segment with the highest plaque area was chosen for quantitative analysis. A total of 116 treated arterial sites were analyzed. Tissue slides were scanned at $\times 4$ magnification with a microscope linked to a charge-coupled device camera (Nikon 104, Duesseldorf, Germany) and analyzed by computer-assisted morphometry (Adobe Photoshop version 5.0, Adobe Systems Inc., San Jose, CA, USA). Intima media ratio was calculated from the measured area of intima and area of media, and lesion area index from the lesion area related to the area of media.

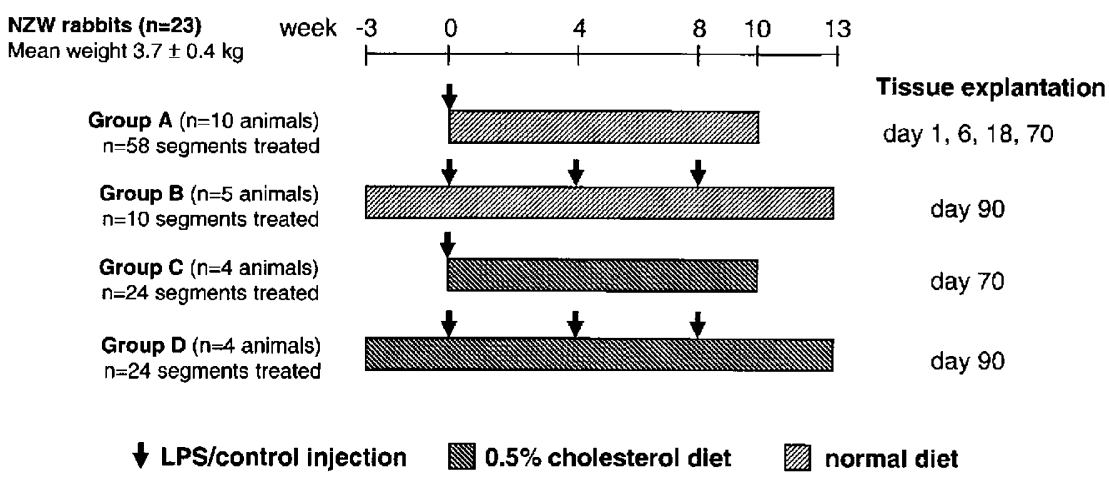

Figure 1 Schematic overview of study groups treated with either single or repeated injections of LPS or sodium chloride (control). Two groups of rabbits (group A, $n=4$ and group B, $n=5$ ) were fed a standard diet, group $C$ animals $(n=4)$ were fed an atherogenic diet supplement including $0.5 \%$ cholesterol for 10 weeks. Four animals (group D) were fed a prolonged cholesterol diet consisting of 16 weeks of feeding. Additional six rabbits on the standard diet were used to test the concentration of LPS and for the assessment of the vessel reaction at early time points. In total, 116 arterial segments were treated and analyzed. 
To determine the cell composition and proliferation activity of lesions, serial sections $(10 \mu \mathrm{m})$ were treated with monoclonal antibodies against markers on rabbit macrophages ${ }^{9}$ (RAM 11, Dako, Hamburg, Germany), against human smooth muscle actin ${ }^{10}$ (HHF35, Enzo Diagnostics, New York, NY, USA), and rabbit lymphocyte CD11a (KEN-11, Pharmingen, Heidelberg, Germany), and detected by an alkaline phosphatase-mounted secondary antibody (Boehringer Mannheim, Mannheim, Germany) or streptavidin-biotin-mounted secondary antibody (Vectastain Laboratories, Burlingame, CA, USA) and counterstained with hematoxylin. Tissues were also stained using a monoclonal antibody against vascular cell adhesion molecule (VCAM)-1 (clone $\mathrm{Rb} 1 / 9$, gift from Professor J Thiery, University Leipzig, Germany) intended to detect the possible expression of VCAM-1 in endothelial cells. All immunohistochemical analyses were controlled using isotype-matched antibodies carried out to prove the specificity of staining.

\section{Evaluation of The Inflammatory Auricular Skin Reaction and Laboratory Analysis}

For evaluation of the auricular skin reaction after perivascular administration of LPS or sodium chloride, the skin reaction was macroscopically examined by measurement of the longitudinal and transverse diameter of the ellipse of developing erythema. The area of the inflammatory reaction was calculated from an equation (ellipse area $=a / 2 \times b /$ $2 \times \pi$ ). Laboratory analyses of the white blood count (WBC) and C-reactive protein (CRP) was performed from blood taken prior to the injection, and at days $2,7,21$ and 70 following injection.

\section{Statistical Analysis}

Results are expressed in means \pm s.e. Intima media ratio and lesion area index between groups were compared by one-factorial analysis of variance using Scheffé's F-test. A level of $P<0.05$ was taken to indicate statistical significance (SPSS release 11.0.1, SPSS Inc., Chicago, IL, USA).

\section{Results}

\section{Clinical and Laboratory Findings}

Perivascular injection of LPS resulted in transient development of erythema at the application site. The peak skin reaction was observed at day 1 after application and differed in size significantly from the site of the control injection $(4.42 \pm 0.52$ vs $0.17 \pm 0.07 \mathrm{~cm}^{2}, P<0.001$, see Figure 2). Mild erythema lasted for 4 days and resolved in all animals. Animals developed a temporary systemic inflammatory reaction with a slightly increased WBC and CRP during the first week after application.

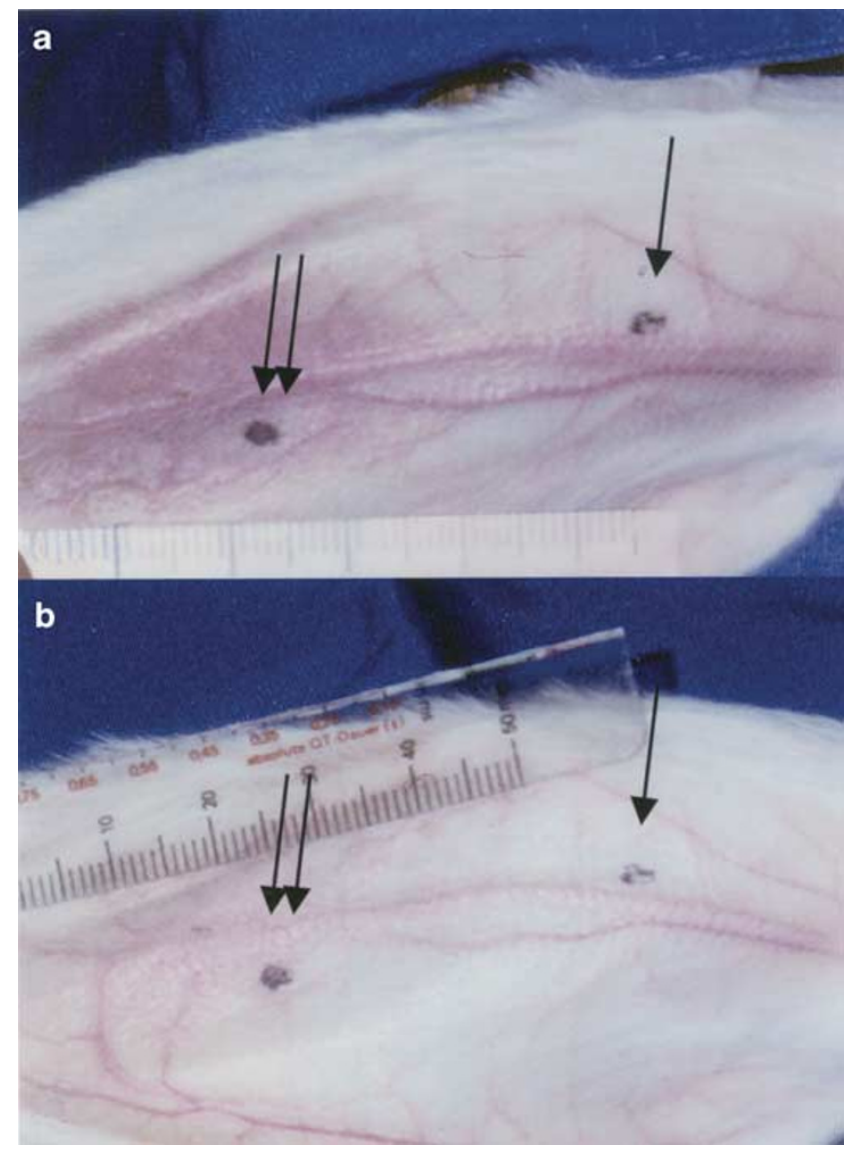

Figure 2 Macroscopic local tissue reaction and distribution after perivascular injection. Transient development of erythema at the application site (rabbit ear); the peak skin reaction was observed at day 1(a) after LPS (double arrows) application and differed in size significantly from the site of the control injection (arrow). Local erythema resolved completely at day $4(\mathbf{b})$.

\section{Effects of LPS on Intima and Lesion Development}

A local inflammatory reaction, with macrophage infiltration, was observed at day 1 around LPStreated arterial vessels, corresponding to the time course of local skin erythema. The presence of macrophages diminished after 6 days. Lymphocytes were absent. Transiently at day 18, significant focal intimal thickening was seen in LPS-treated vessels (intima media ratio $0.077 \pm 0.008$ vs $0.051 \pm 0.005$ in control arteries, $P=0.014$, see Figure 3 ). The thickened intima consisted mostly of vascular smooth muscle cells, inflammatory cells were absent (Figure $5 \mathrm{a}$ and $\mathrm{c}$ ). No intimal thickening was observed in control vessels. At day 90, intimal area returned to normal and was similar in both LPS- and sodium chloride-treated vessels (repeated application, intima media ratio $0.042 \pm 0.008$ vs $0.039 \pm 0.007, P=1.0$, see Figure 3 ).

Cholesterol feeding of the animals induced small atheromatous lesions, but did not lead to enhanced lesion area index compared with 
a

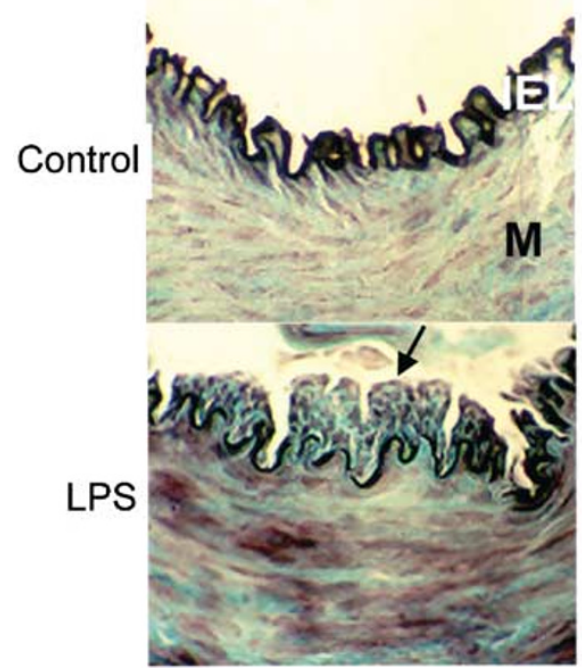

b

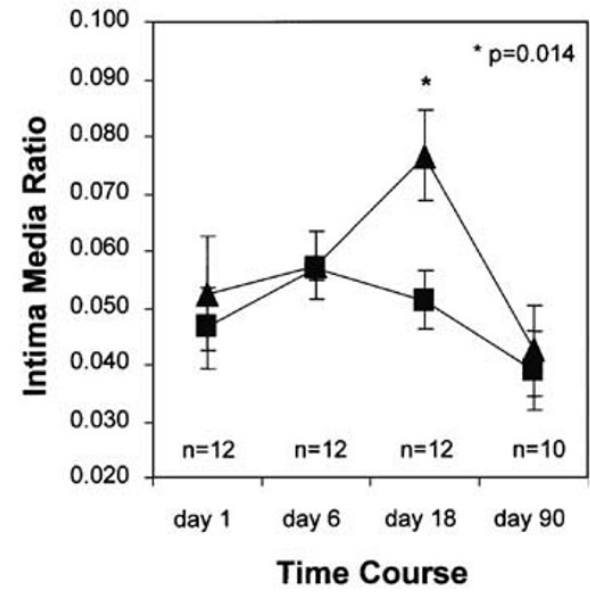

Figure 3 Development of intima thickening after perivascular LPS treatment. In normocholesterolemic rabbits, a transient intimal thickening developed after perivascular LPS injection. (a) Van Giesson's elastic staining demonstrates intimal thickening (arrow) in a LPS-treated vessel compared to control artery. IEL: internal elastic lamina, M: media. (b) Time course of intima media ratio ( \pm s.e.) at days 1, 6, 18 and 90 in normal diet-fed rabbits. Significant increase of intima at day 18 in LPS-treated vessel segments (triangles) vs control arteries (squares, $P=0.014$ ). Note that the level of intima media ratio at day 90 compared to day 1 was lower in both LPS- and controltreated arteries because of the growth of the vessel size.

normocholesterolemic control animals (single control injection, $0.015 \pm 0.006$ vs $0.00 \pm 0.00$, $P=1.0$, Figure 4). Cholesterol feeding combined with repeated local delivery of LPS over a period of 90 days resulted in significant atheromatous lesion induction at sites of injection (lesion area index $0.148 \pm 0.049$ vs $0.008 \pm 0.006$ in control arteries, $P=0.003$, Figure $4 \mathrm{~b}$ ). Lesions consisted mainly of macrophages and smooth muscle cells (Figure 5b and d). The histomorphology of the lesions corresponded to stage II of the Stary classification with predominating macrophages and vascular smooth muscle cells. ${ }^{11}$

In normal diet rabbits $(n=5)$ local perivascular injection of LPS did not result in significant atheromatous lesion formation (lesion area index: 0.00 , intima media ratio $0.04 \pm 0.01$ vs $0.04 \pm 0.007$ in control arteries, $P=1.0$ (Figure $4 \mathrm{a}$ and b). Tables 1 and 2 show comparison of intima media ratio and lesion area index in all treated groups.

\section{Discussion}

Focal intimal thickening and leukocyte migration after local LPS administration have previously been described in a rat model without cholesterol feeding. ${ }^{12}$ This group used an LPS-soaked cotton implanted near femoral arteries, which resulted in focal lesions which were rich in smooth muscle cells and persisted for up to 6 weeks. The present investigation confirms the development of transient intimal thickening 3 weeks after a single local injection of LPS given next to arteries in normocho- lesterolemic rabbits. The dosage of LPS applied in rabbits was only $8 \%$ of the amount applied to the rat model corrected for the body weight, resulting in a minimal systemic inflammatory reaction. Rabbits are more sensitive to LPS compared to rats and the dosage given in the above-described rat experiments leads to septicemia with the upregulation of vascular adhesion molecules and premature death in rabbits as described before for rabbit models of septicemia. ${ }^{8,13}$

At long-term observation up to day 70 , no increased lesions formation was observed in the vessel segments treated with single dosages of LPS in rabbits on a normal diet, or in cholesterol-fed rabbits. Following repeated LPS administrations, however, there was a significant increase in atheromatous lesion formation after 90 days in cholesterolfed rabbits compared with control injected sites. The lesions were identified as stage II of the Stary classification, since macrophages and vascular smooth muscle cells predominated the cellular composition. ${ }^{11}$ Thus, both repeated stimulation by bacterial products and a high-cholesterol diet are important for atheromatous lesion promotion. There was no lesion formation in rabbits fed a normal diet. The plaque-promoting effect following local application was similar to results obtained by Lehr et $a 1^{14}$ using repeated systemic administrations of approximately twice the cumulative dosage of LPS in rabbits, which resulted in increased atherosclerosis compared with control animals under conditions of hypercholesterolemia.

The capacity of production of inflammatory cytokines, for example, interleukin- $1 \alpha$ and $-\beta$, or 

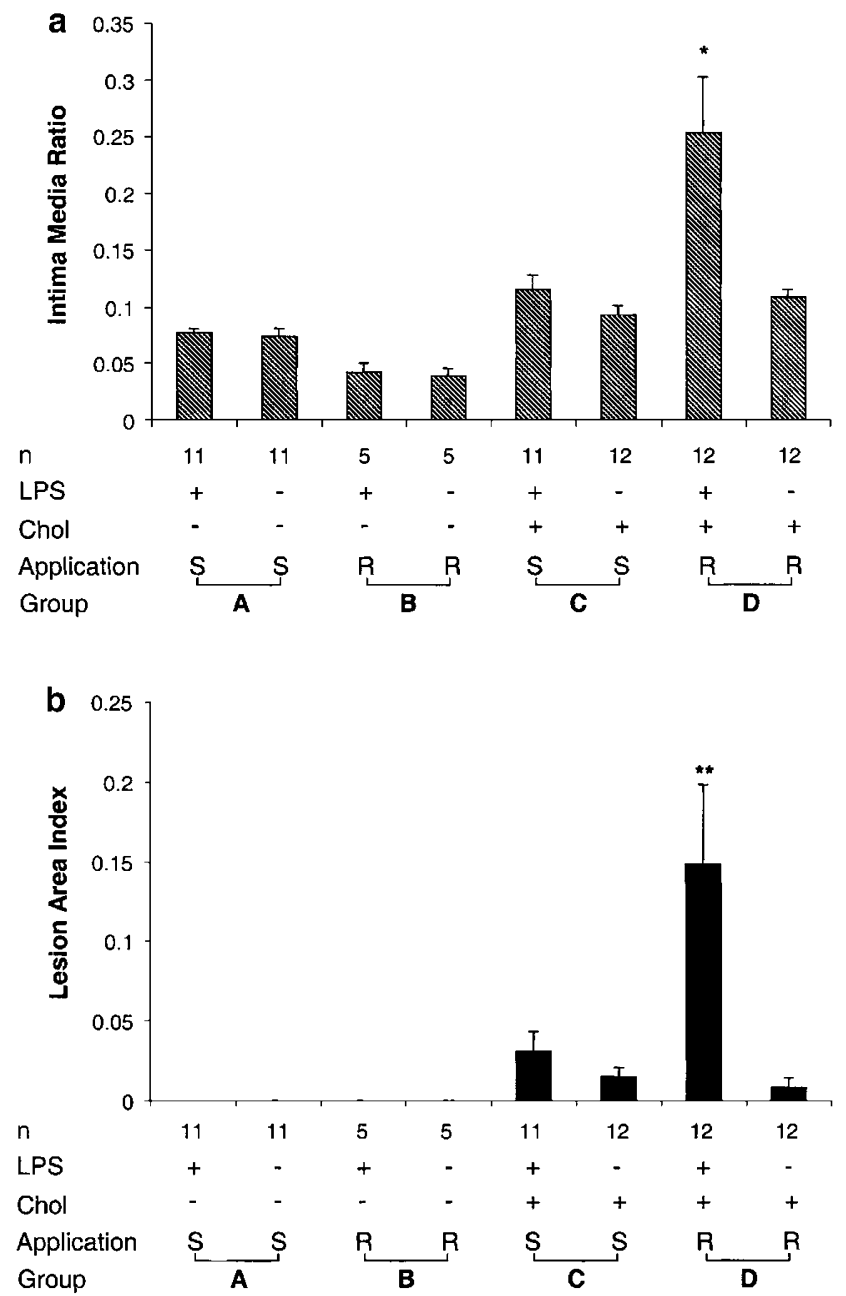

Figure 4 Induction of atheromatous lesion formation by perivascular LPS application. Graph shows intima media ratio (a) and lesion area index (b) in normocholesterolemic (groups A and B) and cholesterol-fed animal groups (groups C and D). In groups A and B (normocholesterolemic, single and repeated application), no difference was observed between LPS and control treatment. Lesion area index in these groups was 0 since no lesions developed. In arterial segments of group D animals (cholesterol-fed, repeated applications), a significant increase in lesion formation was found only in LPS-treated arteries (intima media ratio and lesion area index).

tumor necrosis factor- $\alpha$, in response to LPS is known to be increased in LDL receptor deficiency leading to hypercholesterolemia, ${ }^{6,15-17}$ a mechanism that might contribute to the atheroma-promoting effect of recurrent bacterial infection as demonstrated in our work. Moreover, monocyte recruitment that follows LPS treatment is mediated by macrophage chemoattractant protein-1, resulting in an acceleration of atherogenesis, as demonstrated in an Apo Edeficient mouse model. ${ }^{18}$ LPS injections increase atherosclerotic lesion size supported by a $\mathrm{T}$ helper cell (Th)1 and Th2 T-cell-mediated B-cell activation using an Apo E-deficient mouse model. ${ }^{19}$ Apart from their role in LPS-mediated endothelial damage, ${ }^{20}$ the proinflammatory cytokines increase the expression of adhesion molecules and enhance the uptake of oxidized LDL through increased expression of scavenger receptors. ${ }^{21}$ Bacterial LPS induces tissuefactor expression in lipid-laden macrophages, thus contributing to a higher thrombogeneicity of the atheromatous core, ${ }^{22}$ and increased monocyte and T-cell adhesion via coexpression of heat-shock proteins and adhesion molecules. ${ }^{5}$ Gram-negative LPS (from E. coli as well as $C$. pneumoniae) induces lipid accumulation and foam cell formation from macrophages in vitro, ${ }^{4,23}$ a mechanism that might explain increased lesion formation demonstrated in vivo by our group and others. ${ }^{6,15-17,24}$ Recently, tolllike receptor 4 , the receptor recognizing bacterial LPS, was identified in adventitial fibroblasts and macrophages in human atherosclerotic plaques and was shown to augment neointima formation in a mouse model of adventitial delivery of LPS. ${ }^{25}$ In addition, the oxidative modification of low-density lipoprotein is enhanced by LPS. ${ }^{26}$

The current perivascular inflammation model will allow the assessment of the impact of defined dosages of different bacterial pathogens onto 


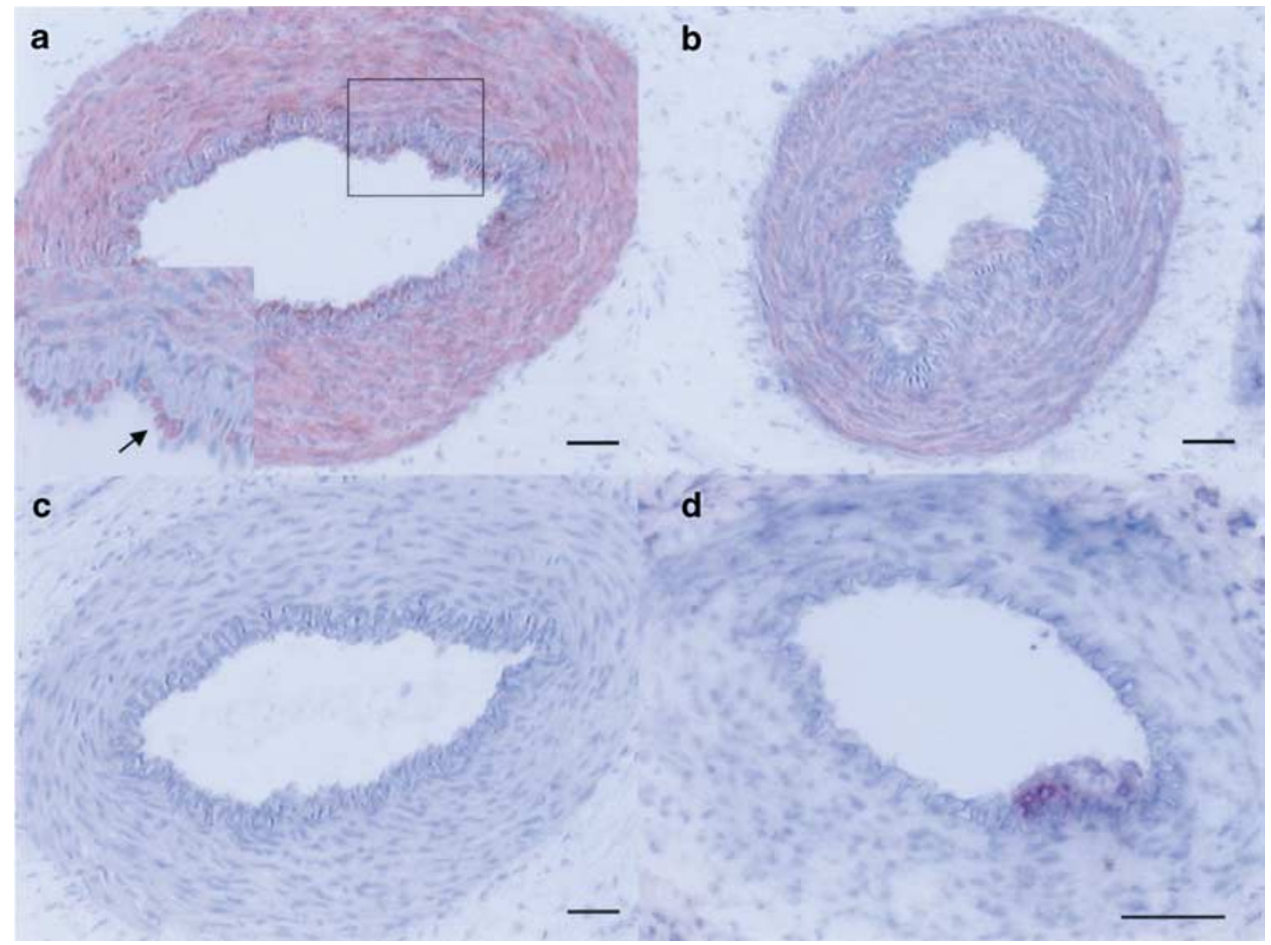

Figure 5 Cellular composition of intima thickening and atheromatous lesions. (a and c) Auricular artery 18 days after perivascular LPS injection. Focal intima thickening consisted mainly of vascular smooth muscle cells as demonstrated by muscle actin staining (a). Inset shows smooth muscle actin-positive cells in detail (arrow). No macrophages were observed in the intima (c). (b and d) Group C (cholesterol-fed) animals with repeated LPS injections. Immunocytochemistry demonstrated the accumulation of smooth muscle cells (b) and macrophages (d) in lesion tissue. Bar represents $50 \mu \mathrm{m}$.

Table 1 Comparison of local perivascular LPS vs control treatment in rabbit arteries

\begin{tabular}{lll}
\hline & \multicolumn{2}{c}{ Intima media ratio } \\
\cline { 2 - 3 } & LPS & Control \\
\hline Group A (chol-, single) $n=(11 / 11)$ & $0.077 \pm 0.005$ & $0.074 \pm 0.007$ \\
Group B (chol-, repeated) $(n=5 / 5)$ & $0.042 \pm 0.008$ & $0.039 \pm 0.007$ \\
Group C (chol+, single) $(n=12 / 12)$ & $0.115 \pm 0.013$ & $0.093 \pm 0.009$ \\
Group D (chol+, repeated) $(n=12 / 12)$ & $0.252 \pm 0.05$ & $0.108 \pm 0.007$ \\
\hline
\end{tabular}

Intima media ratio is expressed in mean \pm s.e. Groups were compared using analysis of variance (Scheffé's test). chol-: normocholesterolemic, chol+: hypercholesterolemic animals. Single: single perivascular injection: repeated; repeated perivascular injections.

Table 2 Comparison of local perivascular LPS vs control treatment in rabbit arteries

\begin{tabular}{|c|c|c|c|}
\hline & \multicolumn{2}{|c|}{ Lesion area index } & \multirow[t]{2}{*}{ P-value } \\
\hline & $L P S$ & Control & \\
\hline Group A (chol-, single) $(n=11 / 11)$ & 0.00 & 0.00 & 1.0 \\
\hline Group B (chol-, repeated) $(n=5 / 5)$ & 0.00 & 0.00 & 1.0 \\
\hline Group C (chol+, single) $(n=12 / 12)$ & $0.031 \pm 0.012$ & $0.015 \pm 0.006$ & 1.01 \\
\hline Group D (chol+, repeated) $(n=12 / 12)$ & $0.148 \pm 0.049$ & $0.008 \pm 0.006$ & 0.003 \\
\hline
\end{tabular}

Lesion area index is expressed in mean \pm s.e. Groups were compared using analysis of variance (Scheffé's test). chol-: normocholesterolemic, chol+: hypercholesterolemic animals. Single: single perivascular injection; repeated: repeated perivascular injections.

predefined sites of the vascular wall in the context of atherogenesis. The model is limited by the artificial way of presenting bacterial pathogens to the vascular wall, since those bacteria that have been shown to be atherogenic (ie C. pneumoniae) are usually transmitted via the respiratory tract. 
In summary, repeated perivascular administration of low-dose LPS in a cholesterol-fed rabbit leads to local lesion formation. This animal model will allow the assessment of the impact of defined dosages of different bacterial pathogens onto the vascular wall in the context of atherogenesis. The atheromatous lesion-promoting effect of repeated perivascular administration of LPS supports the hypothesis that bacterial pathogens may be involved in the response to injury of arteries that results in atherogenesis.

\section{Acknowledgement}

We thank TY Huehns, MRCP, for her help in preparing the manuscript, B Leitermeier and I Plonner for technical assistance. This work was funded in part by the Research Foundation of the Ludwig Maximilian University, Munich (MGE, grant 126). This investigation was presented in part at the Scientific Sessions 2001 of the American Heart Association in Anaheim, CA, USA, November 2001 and at the Scientific Sessions 2002 of the American College of Cardiology in Atlanta, GA, USA.

\section{References}

1 Ross R. Atherosclerosis-an inflammatory disease. N Engl J Med 1999;340:115-126.

2 Ellis RW. Infection and coronary heart disease. J Med Microbiol 1997;46:535-539.

3 Gupta S, Camm AJ. Is there an infective aetiology to atherosclerosis? Drugs Aging 1998;13:1-7.

4 Funk JL, Feingold KR, Moser AH, et al. Lipopolysaccharide stimulation of RAW 264.7 macrophages induces lipid accumulation and foam cell formation. Atherosclerosis 1993;98:67-82.

5 Seitz CS, Kleindienst R, Xu Q, et al. Coexpression of heat-shock protein 60 and intercellular-adhesion molecule-1 is related to increased adhesion of monocytes and $\mathrm{T}$ cells to aortic endothelium of rats in response to endotoxin. Lab Invest 1996;74:241-252.

6 Netea MG, Demacker PN, Kullberg BJ, et al. Increased interleukin-1alpha and interleukin-1beta production by macrophages of low-density lipoprotein receptor knock-out mice stimulated with lipopolysaccharide is CD11c/CD18-receptor mediated. Immunology 1998;95:466-472.

7 Unkelbach K, Gardemann A, Kostrzewa M, et al. A new promoter polymorphism in the gene of lipopolysaccharide receptor CD14 is associated with expired myocardial infarction in patients with low atherosclerotic risk profile. Arterioscler Thromb Vasc Biol 1999;19:932-938.

8 Ikeda N, Mukaida N, Kaneko S, et al. Prevention of endotoxin-induced acute lethality in Propionibacterium acnes-primed rabbits by an antibody to leukocyte integrin beta 2 with concomitant reduction of cytokine production. Infect Immun 1995;63: 4812-4817.
9 Tsukada T, Rosenfeld M, Ross R, et al. Immunocytochemical analysis of cellular components in atherosclerotic lesions. Use of monoclonal antibodies with the Watanabe and fat-fed rabbit. Arteriosclerosis 1986;6:601-613.

10 Skalli O, Ropraz P, Trzeciak A, et al. A monoclonal antibody against alpha-smooth muscle actin: a new probe for smooth muscle differentiation. J Cell Biol 1986;103:2787-2796.

11 Stary HC, Chandler AB, Dinsmore RE, et al. A definition of advanced types of atherosclerotic lesions and a histological classification of atherosclerosis. A report from the Committee on Vascular Lesions of the Council on Arteriosclerosis, American Heart Association. Circulation 1995;92:1355-1374.

12 Prescott MF, McBride CK, Court M. Development of intimal lesions after leukocyte migration into the vascular wall. Am J Pathol 1989;135:835-846.

13 Iiyama K, Hajra L, Iiyama M, et al. Patterns of vascular cell adhesion molecule-1 and intercellular adhesion molecule-1 expression in rabbit and mouse atherosclerotic lesions and at sites predisposed to lesion formation. Circ Res 1999;85:199-207.

14 Lehr HA, Sagban TA, Ihling C, et al. Immunopathogenesis of atherosclerosis: endotoxin accelerates atherosclerosis in rabbits on hypercholesterolemic diet. Circulation 2001;104:914-920.

15 Leirisalo Repo M, Jaattela M, Gylling $\mathrm{H}$, et al. Phagocyte function in familial hypercholesterolaemia: peripheral blood monocytes exposed to lipopolysaccharide show increased tumour necrosis factor production. Scand J Immunol 1990;32:679-685.

16 Netea MG, Demacker PN, Kullberg BJ, et al. Low-density lipoprotein receptor-deficient mice are protected against lethal endotoxemia and severe Gram-negative infections. J Clin Invest 1996;97: 1366-1372.

17 Rovers C, Netea MG, de Bont N, et al. LPS-induced cytokine production and expression of beta2-integrins and CD14 by peripheral blood mononuclear cells of patients with homozygous familial hypercholesterolemia. Atherosclerosis 1998;141:99-105.

18 Aiello RJ, Bourassa PA, Lindsey S, et al. Monocyte chemoattractant protein-1 accelerates atherosclerosis in apolipoprotein E-deficient mice. Arterioscler Thromb Vasc Biol 1999;19:1518-1525.

19 Ostos MA, Recalde D, Zakin MM, et al. Implication of natural killer $\mathrm{T}$ cells in atherosclerosis development during a LPS-induced chronic inflammation. FEBS Lett 2002;519:23-29.

20 Reidy MA, Bowyer DE. Distortion of endothelial repair. The effect of hypercholesterolemia on regeneration of aortic endothelium following injury by endotoxin. Atherosclerosis 1978;29:459-466.

$21 \mathrm{Li} \mathrm{H}$, Freeman MW, Libby P. Regulation of smooth muscle cell scavenger receptor expression in vivo by atherogenic diets and in vitro by cytokines. J Clin Invest 1995;95:122-133.

22 van den Eijnden MM, van Noort JT, Hollaar L, et al. Cholesterol or triglyceride loading of human monocyte-derived macrophages by incubation with modified lipoproteins does not induce tissue factor expression. Arterioscler Thromb Vasc Biol 1999;19:384-392.

23 Kalayoglu MV, Byrne GI. Induction of macrophage foam cell formation by Chlamydia pneumoniae. J Infect Dis 1998;177:725-729. 
$24 \mathrm{Hu} \mathrm{H}$, Pierce GN, Zhong G. The atherogenic effects of chlamydia are dependent on serum cholesterol and specific to Chlamydia pneumoniae. J Clin Invest 1999;103:747-753.

25 Vink A, Schoneveld AH, van der Meer JJ, et al. In vivo evidence for a role of toll-like receptor 4 in the

development of intimal lesions. Circulation 2002;106: 1985-1990.

26 Maziere C, Conte MA, Dantin F, et al. Lipopolysaccharide enhances oxidative modification of low density lipoprotein by copper ions, endothelial and smooth muscle cells. Atherosclerosis 1999;143:75-80. 